\title{
Editorial
}

\section{Editorial: New Publication Mode for "Issues and Trends in Educational Technology"}

\author{
Betül C. Czerkawski \\ Editor, ITET
}

\author{
Max Lieberman \\ Managing Editor, ITET
}

\begin{abstract}
Recognizing that academic culture is adapting to technological affordances, Issues and Trends in Educational Technology will now publish articles as they are approved for inclusion in each issue.
\end{abstract}

Keywords: editorial; open journal systems; publishing frequency; fasterpublication

Anyone who has worked on an academic journal knows how much labor goes into a single issue. This is especially true of first issues. Although the recent inaugural issue of Issues and Trends in Educational Technology was not a long one, we found ourselves immersed so deeply in the task that we were nearly underwater. We formed a strong review board; decided on editorial policies; integrated our website with publication indexes and other online services; and sent out invitations for submissions.

Of all the choices we have made, one of the most important was our publishing frequency. We decided to publish two issues per year, but also to post articles as they become available for publication. The first issue will be complete by the end of June each year, and we will begin publishing our second issue each July.

As you may know, if you frequent the websites of open access journals, ITET uses the Open Journals Systems platform. We receive technical support-and invaluable insight into journal publication. When we asked our librarian colleagues about the possibility of publishing articles as we go, they told us that open access journals are increasingly adopting this model, which provides a quicker publication process for the authors.

Why is this so important to emphasize? As scholars, while we prepare our own manuscripts to submit to various peer-reviewed journals, the submission policies of individual journals can have a big impact on the publication experience. Take our experience with a major educational technology journal, which kept a manuscript under review for more than a year! We received little information on the status of our article, other than notifications when new reviewers were assigned for our paper. Eventually, the editors informed us that a decision would be made soon-an announcement which was followed by an 
additional 6 months of silence. Eighteen months is a long time in a fast-moving field like Educational Technology, and we had to update our article significantly to make it ready for publication.

We hope that our decision to publish as articles become available will help our authors to share truly current research findings. Another implication of this decision is that our current issue will have more additions as we progress through the year, so be sure to check us back when you get a chance.

Another development that we are very excited about is that our first graduate student research paper will be published in this issue. One of the missions of our journal is to provide access to exemplary graduate student work so that students can develop first-hand experience in scholarship and get the encouragement they need in publishing their work. As you can see, graduate student work is presented in a separate section of the Journal, with a distinct and clearly-articulated review process. If you have any distinguished student papers (course projects, papers written as part of a graduate coursework, grants, etc.) that you think would be appropriate for ITET, please submit them. These papers will not go through the regular peer review process, but they will be edited by us. We also encourage your graduate students to send us book, article or technology reviews to be considered for ITET. Since we are an online journal, we support articles accompanied by multimedia including video, interactive figures, software and other non-standard supplementary materials. 